\title{
Etiologic Study of Upper Respiratory Infections of Household Dogs
}

\author{
Masami MOCHIZUKI ${ }^{1,2) *}$, Akiko YACHI ${ }^{1)}$, Takahisa OHSHIMA ${ }^{2)}$, Atsuo $\mathrm{OHUCHI}^{2)}$ and Takuo ISHIDA ${ }^{3)}$ \\ ${ }^{1)}$ Laboratory of Clinical Microbiology, Kyoritsu Seiyaku Corporation, Kudankita 1-12-4, Chiyoda-ku, Tokyo 102-0073, \\ ${ }^{2)}$ Advanced Technology Development Center, Kyoritsu Seiyaku Corporation, Takamihara 2-9-22, Tsukuba, Ibaraki 300-1252 and \\ 3) Akasaka Animal Hospital, Akasaka 4-1-29, Minato-ku, Tokyo 107-0052, Japan
}

(Received 12 December 2007/Accepted 1 February 2008)

\begin{abstract}
Infectious tracheobronchitis (ITB), also known as the kennel cough, is a respiratory syndrome of dogs and usually appears to be contagious among dogs housed in groups. Etiologic agent of ITB is multiple and sometimes complex. In the present study, 68 household dogs showing clinical signs of respiratory infection were examined, and 20 dogs $(29.4 \%)$ were found to be positive for either of following agents. Bordetella bronchiseptica (B.b.) was most frequently detected from nasal and oropharynx sites of 7 dogs (10.3\%). Among the viruses examined, canine parainfluenza virus (CPIV) was detected with the highest frequency (7.4\%). Other pathogens included in the order of frequency group 1 canine coronavirus $(4.4 \%)$, canine adenovirus type $2(2.9 \%)$, group 2 canine respiratory coronavirus $(1.5 \%)$, and canine distemper virus (1.5\%). Only 2 cases showed mixed infections. Neither influenza A virus nor canine bocavirus (minute virus of canines) was found in any dogs examined. These results indicate that both B.b. and CPIV are likely to be the principal etiologic agents of canine ITB in Japan, and they may be considered as the target for prophylaxis by vaccination.

KEY WORDS: Bordetella bronchiseptica, canine parainfluenza virus, canine respiratory coronavirus, infectious tracheobronchitis, kennel cough.
\end{abstract}

Respiratory infection is a common clinical problem of dogs, especially among dogs housed in high density such as in pet shops, breeding and boarding kennels, shelters, research facilities, or veterinary clinics. It is usually diagnosed as "infectious tracheobronchitis (ITB)" or more commonly "kennel cough" when the contagious nature of the disease is apparent $[5,22]$. In most cases the upper respiratory sites are infected with single or multiple agents but the disease is generally not life threatening. Typical clinical features are acute onset of paroxysmal dry coughing and nasal discharge. Affected dogs may recover spontaneously within days or weeks, and a minimal medical treatment is indicated except complicated cases showing severe clinical signs. The ITB, however, sometimes may become enzootic and difficult to be stamped out in the group situation because of repeat introduction of novel infectious agents carried by newcomers to the living environment.

The principal etiologic agents of ITB generally recognized are canine parainfluenza virus (CPIV), canine adenovirus type 2 (CAV-2), and Bordetella bronchiseptica (B.b.). Secondary agents are canine herpesvirus, mammalian reoviruses, CAV type $1(\mathrm{CAV}-1)$, bacteria such as Streptococcus sp., Pasteurella sp., Pseudomonas sp., coliforms and mycoplasmas. Of the secondary agents significant depraving association of Streptococcus equi subsp. zooepidemicus [15] and Mycoplasma cynos [16, 36] was recently described. More recently, some viruses emerged as novel pathogens for canine respiratory tract; group 1 canine coronavirus (CCoV) called "pantropic CCoV" in Italy [11],

\footnotetext{
* Correspondence to: Mochizuki, M., Laboratory of Clinical Microbiology, Kyoritsu Seiyaku Corporation, Kudankita 1-124, Chiyoda-ku, Tokyo 102-0073, Japan.

e-mail:msmmchzk@mb.infoweb.ne.jp
}

group 2 canine respiratory coronavirus $(\mathrm{CRCoV})$ in England [21], and canine influenzavirus closely related to equine influenza A virus H3N8 subtype in the U.S. [19].

In this context an etiologic survey for respiratory infections of household dogs was performed because previous epidemiological studies on ITB in Japan were performed in old days $[1,6,31,37,38]$, and the latest finding is indispensable for planning efficient prophylaxis.

\section{MATERIALS AND METHODS}

Clinical samples: A total of 86 swab samples were taken from 68 household dogs that showed clinical signs of respiratory infection. They were swabbed from 44 oropharynx, 35 nasal cavity, and 7 conjunctival sites, and submitted by animal hospitals in various locations of the country (Aomori, Fukushima, Gunma, Ibaraki, Tokyo, Shizuoka, Aichi, Osaka, Okayama, Nagasaki, and Miyazaki) during a period from June 2006 to May 2007. The ages of the dogs were between 1 month and 14 years, and dogs younger than 6 months old and older than 2 years old made up $72.6 \%$ and $14.5 \%$, respectively. Swabs were placed in $2 \mathrm{~m} l$ of Eagle's minimal essential medium and extracts were clarified by centrifugation at $15,000 \mathrm{rpm}$ for $20 \mathrm{~min}$. The resulting supernatant and pellet were examined for viruses and B.b., respectively.

Detection of viral agents: For virus isolation, the supernatant was inoculated into both Madin-Darby canine kidney (MDCK) and felis cutus whole fetus-4 (fcwf-4) [34] cell cultures. They were examined for cytopathic effect and were blind-passaged further 2 times if necessary. The following viruses were specially examined molecularly; minute virus of canines (MVC), CPIV, CCoV, CRCoV, 
canine distemper virus (CDV), and influenza A virus (IAV).

MVC was examined by the PCR assay described previously [30]. CAV serotype specificity was determined by the hemagglutination-inhibition test described previously [6].

For RNA viruses total RNA was obtained from $140 \mu l$ of the supernatant with a QIAamp Viral RNA Mini Kit (QIAGEN Science). Complementary DNA was synthesized by using 9-mer primer (Takara) and avian myeloblastosis virus reverse transcriptase for reverse transcription-PCR (Transcriptase XL; Takara).

Both the 1st and 2nd PCR assays were used for detection of CPIV. The same primer set, reaction mixture, and PCR protocol were applied for the 1st and 2nd PCR. CPIV T1 strain [6] was used as positive control. Primers were designed from an alignment of the attachment protein gene of simian virus (SV) 5 strain W3A, and the nucleotide sequences of the primers are as follows: HN0915F, 5'ATATGGCGGCGTGATTAAAG-3' (sense orientation; located on the SV5 whole genome sequence at positions 7,498 to 7,517); HN1076R, 5'-TGAATCATTCGATTGCCAAA-3' (antisense orientation; located at positions 7,659 to 7,640 ). A reaction mixture was made by mixing $5.0 \mu l$ of 10 times PCR buffer, $3.0 \mu l$ of $25 \mathrm{mM} \mathrm{MgCl}_{2}, 4.0 \mu l$ of 2.5 $\mathrm{mM}$ dNTP Mixture, $0.5 \mu l$ of each primer $(50 \mathrm{pmol} / \mu l), 0.5$ $\mu l$ of sample DNA, $0.25 \mu l$ of Taq polymerase (1.25 U), and $34.25 \mu l$ of distilled water. Amplification was performed with 30 cycles of denaturation at $94^{\circ} \mathrm{C}$ for $30 \mathrm{sec}$, primer annealing at $55^{\circ} \mathrm{C}$ for $30 \mathrm{sec}$, and extension at $72^{\circ} \mathrm{C}$ for 30 sec. The expected size of the 2 nd PCR product was $162 \mathrm{bp}$.

For detection of CDV, the RT-PCR assay that amplifies a portion of viral hemagglutinin gene was used according to the method described previously [29]. Group $1 \mathrm{CCoV}$ was detected by the Taq Man PCR assay described by Gut et al. [23]. Group $2 \mathrm{CRCoV}$ was detected by the RT-PCR assay that targets the hemagglutinin-esterase gene of group 2 coronavirus by the methods described recently [43].

IAV was examined by a commercial test kit (AI-LISA, Kyoritsu Seiyaku Corporation, Tokyo). The kit was based on nucleic acid sequence-based amplification system [18, 28], which targets the matrix protein gene, and all $\mathrm{H}$ subtypes (H1 to H15) of IAV are detected.

Detection of Bordetella bronchiseptica by PCR assay: The swab pellet was suspended in $600 \mu l$ of distilled water and DNA was extracted with a QIAamp DNA Mini Kit (QIAGEN Science). Strain D-1 of B.b. (ATCC No. 31124) was used as positive reference. A reaction mixture for the PCR was identical to that for CPIV. Amplification protocol was as follows; after denaturing at $94^{\circ} \mathrm{C}$ for $5 \mathrm{~min}$, amplification was followed with 40 cycles of denaturation at $94^{\circ} \mathrm{C}$ for $50 \mathrm{sec}$, primer annealing at $50^{\circ} \mathrm{C}$ for $1 \mathrm{~min}$, and extension at $72^{\circ} \mathrm{C}$ for $1 \mathrm{~min}$.

Two kinds of primer set were used; one was a primer set, Fla2 and Fla4, which amplifies a upstream region of the flagellin structural gene of B.b. as described by Hozbor et al. [25] (PCR/B.b.). The expected size of the PCR product was $237 \mathrm{bp}$. This PCR assay detects DNA from $2 \times 10^{3}$ or more colony-forming unit (CFU) of B.b.. Another set of primers was originally prepared in the present study from an alignment of the outer membrane lipoprotein gene of the genus Bordetella (PCR/B.sp.), and the nucleotide sequences of the primers designed from the B.b. strain RB50 are as follows: B13615F, 5'-ATCGCTGGGATTACCCCTAC3'(sense orientation; located on the B.b. whole genome sequence at positions 13,615 to 13,634$)$; B13930R, 5'TACTTGAGCGGTTCGAAGGT-3' (antisense orientation; located at positions 13,911 to 13,930 ). The expected size of the PCR product was $316 \mathrm{bp}$. This PCR assay detects DNA from $2 \times 10^{2}$ or more CFU of B.b., and may detect other Bordetella species such as B. Pertussis and B. parapertussis in addition to B.b.. Specificity of the PCR assay was determined by sequencing of the PCR product that was applicable for sequence analysis quantitatively as well as qualitatively.

Sequence and phylogenetic analyses: Genetic analysis of the spike protein $(\mathrm{S})$ gene of $\mathrm{CRCoV}$ was performed by the method described recently [43]. Genetyx-Win version 4.0 (Software Development Co., Tokyo) and ClustalX version 2.0 were used to produce multiple alignments and phylogenetic trees [27]. The tree was displayed by using rectangular cladogram option in TreeView 1.6.6 [33].

Nucleotide sequence accession numbers: The nucleotide sequence accession numbers in the GenBank database of the indicated virus and bacterium strains used in this study are as follows: bovine coronavirus (BCoV) strain LY-138 (AF058942); BCoV strain Mebus (BCU00735); CCoV strain 1-71 (AY796289); porcine hemagglutinating encephalomyelitis virus (HEV) strain 67N (AF481863); human coronavirus (HCoV) strain OC43 (L14643); CRCoV strains T101 (A Y 150272), 4182 (DQ682406), 02/005 (AB242262); SV5 strain W3A (AF052755); B.b. strain RB50 (BX640449). The nucleotide sequence data originally reported in this paper will appear in the DDBJ/EMBL/ GenBank nucleotide sequence databases under accession no. AB370269 for the S gene of CRCoV strain 06/075.

\section{RESULTS}

Detection of viruses and clinical feature of the diseased dogs: CPIV was most frequently detected from nasal cavity of 5 dogs $(7.35 \%)$ (Table 1$)$. Two dogs were 2 months old and the others were adult ( 1 year and 7 months, 5 years, and 9 years and 4 months old, respectively). Two dogs were concurrently infected with CAV-2 and CRCoV, respectively. Clinical signs of the dogs infected with CPIV were cough, vomiting, nasal discharge, and fever.

CAV was isolated from the nasal cavity of 2 dogs in the MDCK cell culture and they were found to be CAV-2. Both dogs were 2 months old and showed pyrexia, cough and nasal discharge.

CDV was detected from both oropharynx and conjunctiva of a dog and its age was unknown. The dog was presented with a complaint of general respiratory symptoms, and CDV antigen was positive in the oropharynx sample by a commercial CDV detection kit in the hospital. 
Table 1. Detection of viruses and Bordetella bronchiseptica from dogs showing respiratory signs during a period from June 2006 to May 2007

\begin{tabular}{|c|c|c|c|c|c|c|c|c|c|}
\hline $\begin{array}{l}\text { Swab samples } \\
\text { (sample number) }\end{array}$ & $\mathrm{CAV}^{\mathrm{a})}$ & CPIV & $\mathrm{CCoV}$ & $\mathrm{CRCoV}$ & $\mathrm{CDV}$ & MVC & IAV & Bordetella sp. & B.b. \\
\hline Oropharynx (44) & 0 & 0 & 1 & 0 & 1 & 0 & 0 & 5 & 2 \\
\hline Nasal cavity (35) & 2 & 5 & 2 & 1 & 0 & 0 & 0 & 6 & 5 \\
\hline Conjunctiva (7) & 0 & 0 & 0 & 0 & 1 & 0 & 0 & 0 & 0 \\
\hline Total (86) & 2 & 5 & 3 & 1 & 2 & 0 & 0 & 11 & 7 \\
\hline $\operatorname{Dog}(68)$ & $2(2.9 \%)$ & $5(7.4 \%)$ & $3(4.4 \%)$ & $1(1.5 \%)$ & $1(1.5 \%)$ & 0 & 0 & $10(14.7 \%)$ & $7(10.3 \%)$ \\
\hline
\end{tabular}

a) $\mathrm{CAV}$, canine adenovirus; CPIV, canine parainfluenzavirus; $\mathrm{CCoV}$, group 1 canine coronavirus; $\mathrm{CRCoV}$, group 2 canine respiratory coronavirus; CDV, canine distemper virus; MVC, minute virus of canines; IAV, influenza A virus; B.b., Bordetella bronchiseptica.

Group $1 \mathrm{CCoV}$ was detected from either oropharynx or nasal cavity of 3 dogs. Two dogs were 3 months old and their clinical signs were cough and nasal discharge. The remaining one dog was 1 year old and clinically diagnosed as bronchitis with fever, nasal discharge, cough and sneeze. The hospital medical record of this dog showed isolation of Corynebacterium sp. in the nasal discharge.

Group $2 \mathrm{CRCoV}$ was found in the nasal cavity of a 2 months old puppy, and CPIV was also found in the same sample. The puppy showed both respiratory (mucopurulent nasal discharge and sneeze) and enteric (diarrhea) signs, but no viral agent was found in the rectal sample. Although CRCoV was not recovered in the HRT-18 cell culture from this PCR-positive sample No. 06/075, genetic analysis of S gene was successfully performed. The S gene of No. 06/075 was 4,092 nucleotide long, representing 1,364 predicted amino acids. The nucleotide (amino acid) sequence identities of No. 06/075 S gene for those of CRCoV strains 02/ 005, T101, and 4182 were $99.3 \%$ (98.5\%), 99.0\% (98.5\%), and $98.9 \%(98.3 \%)$, respectively. Figure 1 shows phylogenetic relation of the nucleotide sequence of No.06/075 to other groups 1 and 2 coronaviruses. The closest relationship was to the CRCoV strains, especially to the Japanese CRCoV strain 02/005, forming a different cluster from that of English CRCoV strains.

Neither IAV nor MVC was detected from any samples of 68 dogs.

Detection of Bordetella bronchiseptica and clinical feature of the diseased dogs: Six nasal and 5 oropharynx samples from 10 dogs aged between 2 and 3 months old were regarded as Bordetella sp. positive by the PCR/B.sp. (Fig. 2). Of which, 5 nasal and 2 oropharynx samples of 7 dogs were positive for B.b. specific flagellin structural gene by the PCR/B.b., and in Fig. 3, an example of the PCR results was presented. A common clinical sign of the puppies infected with B.b. was cough and, in the order of frequency, the others included nasal discharge $(71.4 \%)$, fever $(42.9 \%)$, sneeze $(28.6 \%)$, conjunctivitis $(14.3 \%)$, and anorexia $(14.3 \%)$.

\section{DISCUSSION}

Etiology of canine ITB is complex, and CPIV, CAV and B.b. are recognized as major pathogens worldwide $[5,22]$.

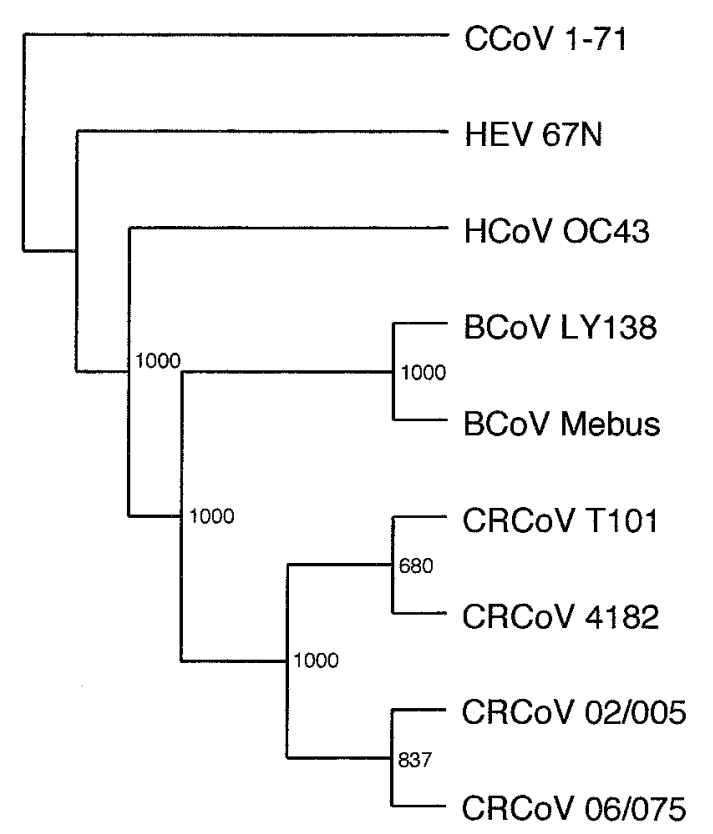

Fig. 1. The phylogenetic tree of the $\mathrm{S}$ gene of $\mathrm{CRCoV}$, other group 2 coronaviruses, and $\mathrm{CCoV}$. $\mathrm{CRCoV}$ strains T101 and 4182, and strains 02/005 and 06/075 are English and Japanese dogs origin, respectively. Each bootstrap value indicates the number of times that each branching was found in 1,000 bootstrap analyses.

Generally ITB may become serious in close confinement where many dogs are housed together. In the present study, the household dogs, either kept a single dog or with a few others, were examined for such well-known ITB pathogens in addition to some novel respiratory pathogenic viruses such as coronaviruses, MVC, and IAV. As a result 20 out of 68 dogs (29.4\%) were positive for either of agents, however etiology of the negative cases remained unknown. It may be necessary to search for an unknown pathogen of canine ITB if hidden.

There was no particular relation between the agent detected and clinical signs appeared. Clinical signs observed for the $20 \mathrm{dogs}$ in the order of frequency were cough, nasal discharge, fever, sneeze, conjunctivitis, and anorexia. Bordetella sp. including B.b. was most frequently 


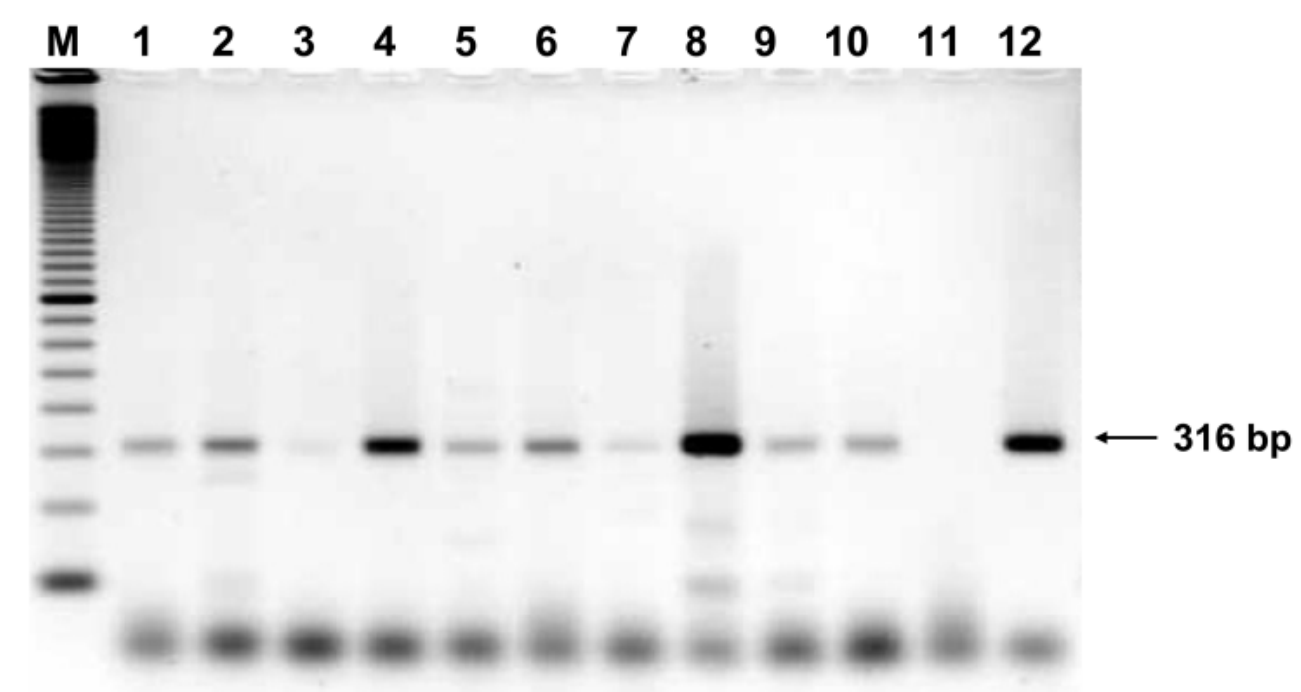

Fig. 2. Detection of Bordetella sp. by the PCR/B. sp. in clinical specimens. A fragment of $316 \mathrm{bp}$ from the outer membrane lipoprotein gene was expected for positive samples. Lanes: M, 100-bp ladder size markers; 1 to 10 , positive clinical samples 06/020, 06/033, 06/039, 06/070, 06/073, 06/081, 06/090, 06/095, 06/098, and 07/007, respectively; 11, negative reference distilled water; 12 , positive reference Bordetella bronchiseptica strain D-1.

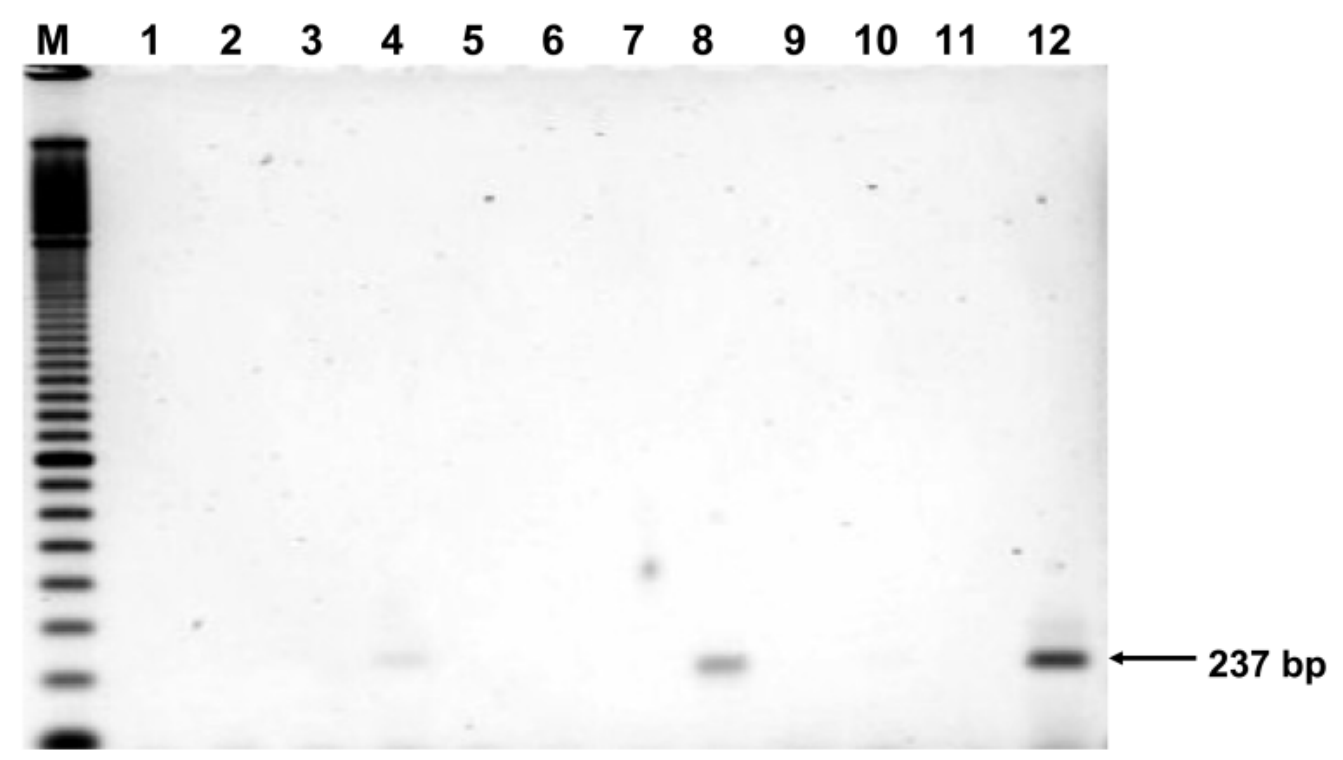

Fig. 3. An example for detection of Bordetella bronchiseptica by the PCR/B.b. in clinical specimens. A fragment of $237 \mathrm{bp}$ from the flagellin structural gene was expected for positive samples. Lanes: M, 100-bp ladder size markers; 4 and 8, positive clinical samples 06/098 and 07/007, respectively; 1 to 3 , 5 to 7,9 , and 10 , negative clinical samples; 11 , negative reference distilled water; 12 , positive reference B.b. strain D-1.

detected, and CPIV, coronaviruses, CAV-2, and CDV followed. The present result was compared with the previous report performed for the dogs in a kennel at Tokyo more than 20 years ago [6]. To our knowledge, this was the only work describing ITB (kennel cough) in Japan. Although some study conditions were fundamentally different between the previous and the present studies, significant agents detected from the diseased dogs are very similar; CAV-2, CPIV and B.b. are prominent. Furthermore, this is almost similar to the etiologic specifications described in the 
previous ITB reports from Western countries [5, 22].

In the present study PCR assay was principally used for examination, and it was strongly expected that molecular method might reveal more concurrent infections than the previous study performed by using the cell culturing method [6]. However, contrary to the prediction, there were only 2 cases where multiple agents were detected; one was both CPIV and CAV-2, and another was both CPIV and CRCoV.

Among the known etiologic agents for canine ITB, B.b. was most frequently detected from the diseased dogs in the present study. Although B.b. was sometimes detected in dogs exhibiting no respiratory symptoms [17], its significance as a pathogen to canine respiratory tract has been established both epidemiologically $[6,7,9,10,17,40]$ and experimentally $[8,39,42]$. Two kinds of the PCR assay were applied here and the PCR/B.sp. for detection of Bordetella sp. did show higher positive rate than the PCR/B.b. (Table 1). Thus, it is natural to regard that Bordetella sp. other than B.b. had also existed in the samples if the result is understood as it is. On the other hand, since the PCR/B.b. is about 10-times less sensitive than the PCR/B.sp., it is another possibility that the positive results by the PCR/B.sp. might originate from the B.b. present.

The pathogen was found mainly from puppies aged between 2 and 3 months old in the present study. One possible explanation is that they became sick soon after their introduction to the new owner's home because they had been exposed to the pathogens during group breeding. One exceptional finding was CPIV and it was detected also from the aged dogs. Of 5 CPIV-positive dogs 3 dogs were older than 1.5 years old. CPIV might widely spread in the dog society when compared with other ITB agents.

In the present study both groups 1 and 2 coronaviruses were detected from respiratory sites of the diseased dogs. Group $1 \mathrm{CCoV}$ has been recognized as a mild enteric pathogen for dogs [3]. Thus it is a likely explanation that such positive reactions originate from the contamination from the digestive tract. While $\mathrm{CCoV}$ is a well-known virus species that mutates frequently [35], and for an example of pathogenic mutant, a novel pantropic $\mathrm{CCoV}$ was recently discovered [11]. Thus further studies to elucidate pathogenic potential of $\mathrm{CCoV}$ against canine respiratory tract may be necessary. Group $2 \mathrm{CRCoV}$, recently isolated in England as a new ITB pathogen closely related to bovine coronavirus [20], was again found to be associated with the diseased puppy in the present study. CRCoV infection was already confirmed in Japanese domestic dogs both seroepidemiologically [26] and molecular epidemiologically [43]. The present No. 06/075 strain is the second CRCoV strain discovered in Japan and phylogenetic analysis of the $\mathrm{S}$ gene of No. 06/075 together with those of the previous English and Japanese strains suggest there may be a regional lineage in evolution of CRCoV (Fig. 1).

Neither IAV nor MVC was associated with the clinical cases in the present study. Although IAV is a well-known respiratory pathogen for humans, horses, pigs and avian species, the previous studies suggested less or no clinical signif- icance of IAV as a pathogen in canine ITB though dogs can be infected with several IAV subtypes [4]. However, recent canine IAV epidemic in both the U.S. [19, 41] and England [32], and severe dog cases entangled in the highly pathogenic avian influenza outbreaks of domestic fowls in the Southeast Asia region [12] drew our re-attention to IAV as a potential pathogen for respiratory tract of dogs. As a result no evidence for IAV participation in the present cases was obtained, suggesting that IAV is not an important pathogen for dogs at the ordinary time in the environment where such specific IAV has not broken out.

Bocavirus is a new genus assigned into Parvovirus family, and bovine parvovirus and canine parvovirus 1, originally named as MVC, have been classified. MVC is a pathogen basically for canine fetus and newborn puppy and causes both respiratory and enteric diseases in the puppy $[13,14]$. Recently human bocavirus has been found to be associated with human lower respiratory diseases [2]. Thus, by way of precaution, its association with the canine ITB was determined in the present study since it was revealed that MVC infection was widespread among Japanese domestic dogs [24, 30]. However no evidence for MVC participation in the respiratory disease was obtained, suggesting either minimal or no pathogenic potential for elder dogs.

In summary, it was confirmed that both B.b. and CPIV are likely the principal infectious etiologic agents of respiratory disease of the household dog in Japan. CAV-2 had the second importance. These findings were in accordance with the kennel cough study performed two decades ago in Tokyo. In Japan, Bordetella vaccine has never been clinically used for dogs so far and CPIV vaccine has been available only as a fraction of the combined parenteral vaccine. This implies that some other prophylaxis measures such as the introduction of intranasal kennel cough vaccines containing B.b. and CPIV may be needed for more efficacious ITB control in the susceptible dog groups.

ACKNOWLEDGEMENTS. The authors wish to express our gratitude to the following practicing veterinarians for sample submission; Drs. Yasuhiko Tsuchida, Atsushi Kawamata, Goro Kurita, Moriyasu Kishi, Akiko Shibanai, Naomi Honma, Norihiro Yoshimura, Kouji Nishida, Tetsuya Shimoda, Hideo Sakai, and Kiyotaka Kushima. CPIV T1 strain was provided by Dr. Yukinobu Tohya of the University of Tokyo.

\section{REFERENCES}

1. Ajiki, M., Takamura, K., Hiramatsu, K., Nakai, M., Sasaki, N. and Konishi, S. 1982. Isolation and characterization of parainfluenza 5 virus from a dog. Jpn. J. Vet. Sci. 44: 607-618.

2. Allander, T., Tammi, M. T., Ericksson, M., Bjerkner, A., Tiveljung-Lindell, A. and Andersson, B. 2005. Cloning of a human parvovirus by molecular screening of respiratory tract samples. Proc. Natl. Acad. Sci. U.S.A. 102: 12891-12896.

3. Appel, M. 1987. Canine coronavirus. pp. 115-122. In: Virus Infections of Vertebrates (Appel, M. J. ed.), Elsevier, Amsterdam. 
4. Appel, M. 1987. Influenza viruses. pp. 163-164. In: Virus Infections of Vertebrates (Appel, M. J. ed.), Elsevier, Amsterdam.

5. Appel, M. and Binn, L. N. 1987. Canine infectious tracheobronchitis. Short review: kennel cough. pp. 201-211. In: Virus Infections of Vertebrates (Appel, M. J. ed.), Elsevier, Amsterdam.

6. Azetaka, M. and Konishi, S. 1988. Kennel cough complex: Confirmation and analysis of the outbreak in Japan. Jpn. J. Vet. Sci. 50: 851-858.

7. Bemis, D. A., Carmichael, L. E. and Appel, M. J. G. 1977. Naturally occurring respiratory disease in a kennel caused by Bordetella bronchiseptica. Cornell Vet. 67: 282-293.

8. Bemis, D. A., Greisen, H. A. and Appel, M. J. G. 1977. Pathogenesis of canine bordetellosis. J. Infect. Dis. 135: 753-762.

9. Binn, L. N., Eddy, G. A., Lazar, E. C., Helms, J. and Murnane, T. 1967. Viruses recovered from laboratory dogs with respiratory disease. Proc. Soc. Exp. Biol. Med. 126: 140-145.

10. Binn, L. N., Lazar, E. C., Rogul, M., Shepler, V. M., Swango, L. J., Claypoole, T., Hubbard, D. W., Asbill, S. G. and Alexander, A. D. 1968. Upper respiratory disease in military dogs: bacterial, mycoplasma, and viral studies. Am. J. Vet. Res. 29: 1809-1815.

11. Buonavoglia, C., Decaro, N., Martella, V., Elia, G., Campolo, M., Desario, C. and Tempesta, M. 2006. Canine coronavirus highly pathogenic for dogs. Emerg. Infect. Dis. 12: 492-494.

12. Butler, D. 2006. Thai dogs carry bird-flu virus, but will they spread it? Nature 439: 773.

13. Carmichael, L. E., Schlafer, D. H. and Hashimoto, A. 1991. Pathogenicity of minute virus of canines (MVC) for the canine fetus. Cornell Vet. 81: 151-171.

14. Carmichael, L. E., Schlafer, D. H. and Hashimoto, A. 1994. Minute virus of canines (MVC, canine parvovirus type-1): pathogenicity for pups and seroprevalence estimate. J. Vet. Diagn. Invest. 6: $165-174$.

15. Chalker, V. J., Brooks, H. W. and Brownlie, J. 2003. The association of Streptococcus equi subsp. zooepidemicus with canine infectious respiratory disease. Vet. Microbiol. 95: 149156.

16. Chalker, V. J., Owen, W. M., Paterson, C., Barker, E., Brooks, H., Rycroft, A. N. and Brownlie, J. 2004. Mycoplasmas associated with canine infectious respiratory disease. Microbiology 150: 3491-3497.

17. Chalker, V. J., Toomy, C., Opperman, S., Brooks, H. W., Ibuoye, M. A., Brownlie, J. and Rycroft, A. N. 2003. Respiratory disease in kenneled dogs: serological responses to Bordetella bronchiseptica lipopolysaccharide do not correlate with bacterial isolation or clinical respiratory symptoms. Clin. Diagn. Lab. Immunol. 10: 352-356.

18. Compton, J. 1991. Nucleic acid sequence-based amplification. Nature 50: 91-92.

19. Crawford, P. C., Dubovi, E. J., Castleman, W. L., Stephenson, I., Gibbs, E. P. J., Chen, L., Smith, C., Hill, R. C., Ferro, P., Pompey, J., Bright, R. A., Media, M.-J., Influenza Genomics Group, Johnson, C. M., Olsen, C. W., Cox, N. J., Klimov, A. I., Katz, J. M. and Donis, R. O. 2005. Transmission of equine influenza virus to dogs. Science 310: 482-485.

20. Erles, K., Shiu, K.-B. and Brownlie, J. 2007. Isolation and sequence analysis of canine respiratory coronavirus. Virus Res. 124: 78-87.

21. Erles, K., Toomey, C., Brooks, H. W. and Brownlie, J. 2003. Detection of a group 2 coronavirus in dogs with canine infectious respiratory disease. Virology 310: 216-223.
22. Ford, R. B. 2006. Canine infectious tracheobronchitis. pp. 54 61. In: Infectious Diseases of the Dog and Cat, 3rd ed. (Greene, C. E. ed.), Saunders Elsevier, St. Louis.

23. Gut, M., Leutenegger, C. M., Huder, J. B., Pedersen, N. C. and Lutz, H. 1999. One-tube fluorogenic reverse transcriptionpolymerase chain reaction for the quantitation of feline coronaviruses. J. Virol. Methods 77: 37-46.

24. Hashimoto, A., Takiguchi, M., Hirai, K., Kida, H. and Carmichael, L. E. 2001. A serological survey of minute virus of canines (MVC; Canine parvovirus type-1) in dogs in the Tokai area of Japan. Jpn. J. Vet. Res. 49: 249-253.

25. Hozbor, D., Fouque, F. and Guiso, N. 1999. Detection of Bordetella bronchiseptica by the polymerase chain reaction. Res. Microbiol. 150: 333-341.

26. Kaneshima, T., Hohdatsu, T., Satoh, K., Takano, T., Motokawa, K. and Koyama, H. 2006. The prevalence of a group 2 coronavirus in dogs in Japan. J. Vet. Med. Sci. 68: $21-$ 25.

27. Larkin, M. A., Blackshield, G., Brown, N. P., Chenna, R., McGettigan, P. A., McWilliam, H., Valentin, F., Wallace, I. M., Wilm, A., Lopez, R., Thompson, J. D., Gibson, T. J. and Higgins, D. C. 2007. Clustal W and Clustal X version 2.0. Bioinfomatics 23: 2947-2948.

28. Lau, L. T., Banks, J., Aherne, R., Brown, I. H., Dillon, N., Collins, R. A., Chan, K. Y., Fung, Y. W., Xing, J. and Yu, A. C. 2004. Nucleic acid sequence-based amplification method to detect avian influenza virus. Biochem. Biophys. Res. Commun. 313: 336-342.

29. Mochizuki, M., Hashimoto, M., Hagiwara, S., Yoshida, Y. and Ishiguro, S. 1999. Genotypes of canine distemper virus determined by analysis of the hemagglutinin genes of recent isolates from dogs in Japan. J. Clin. Microbiol. 37: 2936-2942.

30. Mochizuki, M., Hashimoto, M., Hajima, T., Takiguchi, M., Hashimoto, A., Une, Y., Roerink, F., Ohshima, T., Parrish, C. R. and Carmichael, L. E. 2002. Virologic and serologic identification of minute virus of canines (canine parvovirus type 1) from dogs in Japan. J. Clin. Microbiol. 40: 3993-3998.

31. Motohashi, T. and Tajima, M. 1966. Isolation of a herpes virus from a diseased adult dog in Japan. Jpn. J. Vet. Sci. 28: $307-$ 314.

32. Newton, R., Cooke, A., Elton, D., Bryant, N., Rash, A., Bowman, S., Blunden, T., Miller, J., Hammond, T.-A., Camm, I. and Day, M. 2007. Canine influenza virus: cross-species transmission from horses. Vet. Rec. 161: 142-143.

33. Page, R. D. M. 1996. TREEVIEW: An application to display phylogenetic trees on personal computers. Comput. Appl. Biosci. 12: 357-358.

34. Pedersen, N. C., Boyle, J. F. and Floyd, K. 1981. Infection studies in kittens, using feline infectious peritonitis virus propagated in cell culture. Am. J. Vet. Res. 42: 363-367.

35. Pratelli, A. 2006. Genetic evolution of canine coronavirus and recent advances in prophylaxis. Vet. Res. 37: 191-200.

36. Rycroft, A. N., Tsounakou, E. and Chalker, V. 2007. Serological evidence of Mycoplasma cynos infection in canine infectious respiratory disease. Vet. Microbiol. 120: 358-362.

37. Shirota, K., Azetaka, M. and Fujiwara, K. 1980. A case of canine respiratory adenovirus infection associated with distemper. Jpn. J. Vet. Sci. 42: 265-270.

38. Takamura, K., Ajiki, M., Hiramatsu, K., Takemitsu, S., Nakai, M. and Sasaki, N. 1982. Isolation and properties of adenovirus from canine respiratory tract. Jpn. J. Vet. Sci. 44: 355-357.

39. Thompson, H., McCandlish, I. A. P. and Wright, N. G. 1976. Experimental respiratory disease in dogs due to Bordetella 
bronchiseptica. Res. Vet. Sci. 20: 16-23.

40. Tischler, S. A. and Hill, J. R. 1977. Kennel cough syndrome in a pet store. J. Am. Anim. Hosp. Assoc. 13: 342-348.

41. Von Grotthuss, M. and Rychlewski, L. 2006. Influenza mutation from equine to canine. Science 311: 1241.

42. Wright, N. G., Thompson, H., Taylor, D. and Cornwell, H. J.
C. 1973. Bordetella bronchiseptica: a re-assessment of its role in canine respiratory disease. Vet. Rec. 93: 486-487.

43. Yachi, A. and Mochizuki, M. 2006. Survey of dogs in Japan for group 2 canine coronavirus infection. J. Clin. Microbiol. 44: $2615-2618$. 